\title{
Comunicación de riesgo y sistemas de información en la Web: cinco modelos
}

\author{
Por Carles Pont-Sorribes, Lluís Codina y Rafael Pedraza-Jimenez
}

Resumen: Las administraciones públicas de los países avanzados están llevando a cabo iniciativas para gestionar la información y la comunicación de riesgo y emergencias mediante sitios web concebidos y diseñados para ello. Estos sitios están pensados para facilitar información a los ciudadanos en caso de emergencias, pero también contienen información útil para los expertos y las autoridades. En este trabajo, y a la luz de la legislación española sobre emergencias, se comparan los sitios de la administración autonómica catalana y del gobierno de España con los sitios de tres países de referencia: Estados Unidos, Francia y Reino Unido. Al mismo tiempo se propone una metodología simple para llevar a cabo una comparación que permita extraer conclusiones y plantear recomendaciones en un aspecto

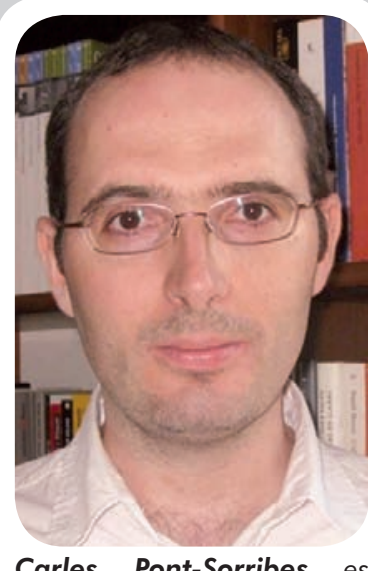

Carles Pont-Sorribes es profesor del departamento de comunicación de la Universitat Pompeu Fabra (UPF) de Barcelona, doctor en comunicación social por la UPF, licenciado en periodismo y diplomado en ciencias de la educación. Investiga sobre la comunicación de riesgo y crisis, así como en los ámbitos de la comunicación política y la opinión publica, y el periodismo local e histórico. Forma parte de los grupos de investigación GRP y Unica de la UPF.

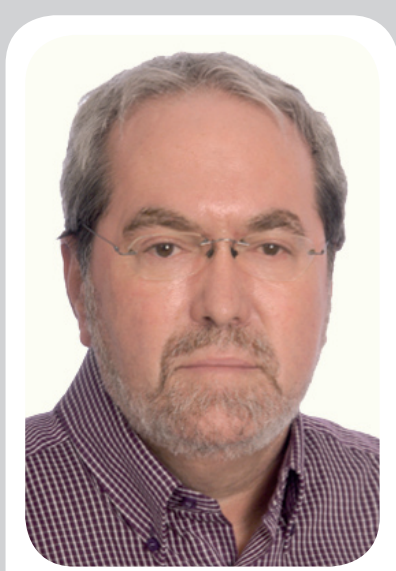

Lluís Codina es profesor titular de la UPF y director de la Unidad de soporte a la calidad y a la innovación docente (Usquid) de la Fac. de Comunicación. Imparte docencia en periodismo y comunicación audiovisual y participa en programas de máster y doctorado en UPF, UB y UPV. Sus intereses se orientan a temas como Web 2.0, Web semántica, sistemas de información documental, documentación periodística... Es fundador y codirector del Máster en documentación digital y del anuario Hipertext.net.

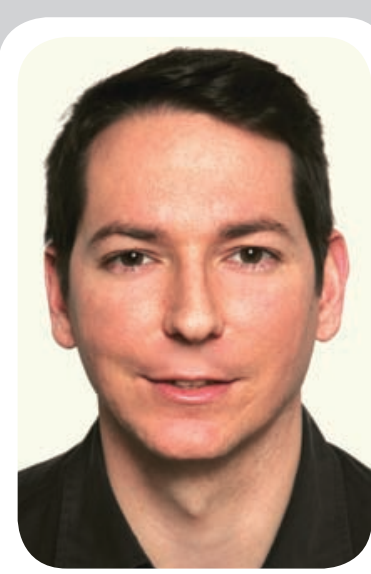

Rafael Pedraza-Jimenez es miembro del grupo de investigación DigiDoc y profesor del área de biblioteconomía y documentación en la Universitat Pompeu Fabra de Barcelona. Imparte docencia en las titulaciones de comunicación audiovisual y periodismo, y en diversos masters, como el Máster online en documentación Digital. Sus principales líneas de investigación se orientan a los campos de la Web semántica y la recuperación de información.

de la gestión de la información que puede resultar clave para salvar bienes materiales y vidas humanas.

Palabras clave: Comunicación de riesgo, Emergencias, Sitios web, Sistemas de información, Catalunya, España, Estados Unidos, Reino Unido, Francia.

\section{Title: Risk communication and web-based information systems: five models}

Abstract: Public administration in the developed countries is designing information initiatives to manage risk information using web-based systems. These sites are intended to deliver information to citizens, but also have valuable information for experts and authorities. Five web sites are compared and a simple methodology is provided to extract conclusions and propose recommendations in an information management dimension that is key to saving not only property but human lives as well.

Keywords: Risk communication, Emergencies, Web sites, Information systems, Catalonia, Spain, United Estates, United Kingdom, France.

Pont-Sorribes, Carles; Codina, Lluís; Pedraza-Jimenez, Rafael. “Comunicación de riesgo y sistemas de información en la Web: cinco modelos”. El profesional de la información, 2009, julio-agosto, v. 18, n. 4, pp. 389-397.

DOI: 10.3145/epi.2009.jul.05

\section{Problema de investigación}

El riesgo, las crisis o las situaciones de emergencia son motivo de una creciente atención tanto por parte de los investigadores sociales como en el campo de la información y la comunicación. Casi todos los protocolos de emergencia incluyen el uso de sistemas de informa- ción que ayuden a los ciudadanos a afrontar tales situaciones. Y junto a los medios de comunicación tradicionales como la prensa escrita, la radio y la televisión, la Web está to- 
mando un protagonismo central en el ecosistema de la comunicación social de buena parte del planeta.

\section{"Las consecuencias de las situaciones de emergencia podrían reducirse con un mejor uso de los sistemas de información"}

Por esta razón, en este artículo partimos del siguiente problema de investigación: ¿hasta qué punto las administraciones hacen un uso adecuado de los sistemas de información, sobre todo de aquellos basados en web en la difusión de información en episodios de emergencias?

Un propósito adicional de este trabajo ha consistido en la elaboración de una propuesta metodológica que permita evaluar la calidad de los sitios web de emergencias ${ }^{1} \mathrm{y}$ protección civil con el fin de contribuir a su mejora. Los componentes de esta propuesta se han obtenido de parámetros universalmente aceptados como propios de sitios web de calidad, como la accesibilidad, la visibilidad, la corrección del código fuente y la adecuación del contenido a las mejoras prácticas internacionales.

Se trata de un problema fundamental puesto que las consecuencias que pueden tener las situaciones de emergencia, no solamente en daños materiales, sino también en pérdida de vidas humanas, podrían eventualmente reducirse con un uso adecuado de los sistemas de información.

Nuestra hipótesis de partida es que en general las administraciones autonómicas y central no explotan todo el potencial de la Web en la comunicación de emergencias.

Una de las razones es que una parte de la legislación, la normativa y los protocolos públicos han quedado obsoletos por el paso del tiempo o por la falta de adaptación a las nuevas posibilidades que permite la técnica. Más concretamente, los responsables de la protección civil han tendido a ignorar las ventajas de la Web.

\section{Metodología}

Para llevar a cabo este artículo se ha estudiado la legislación y el tratamiento de la comunicación de riesgo y de emergencias del " $D e$ partament d'Interior, Relacions Institucionals i Participació" de la Generalitat de Catalunya y del "Ministerio del Interior" del Gobierno de España. Así mismo se ha hecho un estudio de las experiencias existentes, así como de la normativa sobre administraciones públicas y gestión de emergencias de Estados Unidos, Francia, Reino Unido y UE: Federal Emergency Management Agency (FEMA/ EUA), Direction de la Défense et de la Sécurité Civiles (Ministère de l'Intérieur/Francia), Civil Contingencies Secretariat (The Cabinet Office/Reino Unido), The International Civil Defence Organisation (ICDO) y Comisión Europea (Unión Europea).
Se ha realizado un análisis comparativo de las webs de emergencias de Catalunya (como ejemplo representativo de una comunidad con competencias en la gestión de situaciones de riesgo y crisis), España, Francia, Reino Unido y Estados Unidos de América.

\section{Marco teórico}

La noción de comunicación de riesgo evoluciona a partir de los 80 s, sobre todo para dar una respuesta integral a su gestión. Hay una corriente destinada a transmitir información a la población y otras que tratan de un proceso más general, como las que se proponen desde algunos gobiernos, empresas y estamentos internacionales: Unión Europea, OCDE o la International Organisation Standardization (ISO).

La crisis de confianza de la opinión pública con las instituciones, el incremento del conocimiento científico sobre temas básicos como el medio ambiente, o el aumento de responsabilidades antropogénicas en el origen de grandes crisis, han motivado una alta atención sobre la minimización y mitigación de los riesgos. La implementación de sistemas de control o la proliferación de firmas de certificación de calidad son constantes en nuestros días.

Uno de los estamentos internacionales que marcó un punto y aparte en la concepción de la comunicación del riesgo fue el US National Research Council con el informe Improving risk communications, que lo define como: "un proceso

\begin{tabular}{|l|l|}
\hline \multicolumn{1}{|c|}{ País } & \multicolumn{1}{c|}{ Web } \\
\hline España & http://www.inforiesgos.es \\
\hline - Cataluña & http://www.gencat.cat/interior/emergencies/index.htm \\
\hline Estados Unidos de América & $\begin{array}{l}\text { http://www.ready.gov (versión en inglés) } \\
\text { http://www.listo.gov (versión en castellano) }\end{array}$ \\
\hline Francia & http://www.prim.net \\
\hline Reino Unido & $\begin{array}{l}\text { http://www.direct.gov.uk/en/Governmentcitizensandrights/Dealingwithemergencies/ } \\
\text { Preparingforemergencies/index.htm }\end{array}$ \\
\hline
\end{tabular}


interactivo de intercambio de información y de opiniones entre individuos, grupos e instituciones, que a menudo implica mensajes múltiples sobre la naturaleza del riesgo y otros mensajes, no estrictamente sobre riesgo, los cuales expresan preocupaciones, opiniones o reacciones a los mensajes de riesgo o a las disposiciones legales e institucionales de la gestión del riesgo".

Es necesario diferenciar la comunicación de riesgo de otras ramas teóricas parecidas pero que tienen finalidades diferentes. Así, debe ser distinguida de líneas sectoriales que interesan para identificar conflictos parciales, según Farré (2005), como la información de crisis, que se ocupa de los protocolos de actuación que deben seguirse en situaciones planificadas y predefinidas, o la comunicación de crisis, que trata, fundamentalmente, de las relaciones entre la comunicación empresarial y los medios de comunicación social.

\section{"La comunicación de riesgo incluye: el análisis de los efectos, del contenido, de la persuasión y de los actores"}

La comunicación de riesgo como proceso incluye cuatro perspectivas: análisis de los efectos, del contenido, de la persuasión y de los actores. Estos actores son: comunidades afectadas, autoridades públicas, profesionales de la industria, expertos científicos y técnicos, organizaciones civiles y medios de comunicación. El último punto es en el que nos fijaremos en este artículo. Un estudio de Graham Murdock, Tom Horlick-Jones y Judith Petts da algunas pautas sobre las razones del cambio en la comunicación de riesgo, que en parte co- inciden con las que ya han quedado apuntadas en apartados anteriores de este texto.

Los autores británicos evidencian que las tecnologías de la información y las comunicaciones (TIC) deben ser entendidas como una oportunidad más que como un contratiempo, porque la opinión pública accede mayoritariamente al espacio público no solamente mediante la radio, la prensa y la televisión sino también desde internet y teléfono.

La opinión pública, ante riesgos que son significativos, quiere ser atendida (Murdock et al., 2001), es decir, quiere que se le facilite la toma de decisiones sobre si hace falta comprar cierta comida o no, o usar determinados medios de transporte o no. Este proceso de racionalización obliga a las autoridades a extraer interpretaciones y fuentes múltiples de información (Murdock et al., 2001: 91). En consecuencia, la comunicación recíproca entre los diferentes actores que interaccionan en el ámbito de la gestión del riesgo permite una mayor efectividad (Bennet; Calman, 1999).

La intervención de la WWW en este proceso comunicativo tiene un gran protagonismo, y se posiciona como un recurso imprescindible junto a los otros sistemas de información y comunicación.

La facilidad con la que la opinión pública puede tener acceso a la toma de decisiones que apunta Murdock sobre cómo afrontar una emergencia hace de la Red un excepcional repositorio de información que se convierte en crucial en caso de crisis. La mayoría de las administraciones de los países más desarrollados del mundo han depositado en la Web gran parte de la transmisión de información de riesgo, una responsabilidad que años atrás sólo recaía en los medios de comunicación de masas convencionales.

\section{Resultados}

\subsection{Gestión de las emergencias en España}

La creación de la Dirección General de Protección Civil se materializó en 1980 mediante el Real decreto $1547 / 1980$ y fue el embrión de lo que hoy se denomina Sistema Nacional de Protección Civil, que depende del Ministerio del Interior $\mathrm{y}$ en el que desempeñan un papel destacado las comunidades autónomas y las administraciones locales. La normativa básica del Estado para la gestión de emergencias es la Ley de Protección Civil 21/1985.

Esta legislación, que ha recibido diferentes modificaciones mediante reales decretos, tiene como finalidad "el estudio y la prevención de las situaciones de riesgo grave, catástrofe o calamidad pública y la protección de personas y bienes en caso de que estas situaciones se produzcan". Una de las reformas legislativas más importantes fue la aprobación, en 1992, de la Ley Básica de Protección Civil, que pretende integrar los planes de las diferentes administraciones que hay en el Estado español. Esta normativa establece la planificación de las emergencias a partir de planes territoriales (en función de si afectan a todo el Estado, a una comunidad autónoma, a un municipio o a más de un municipio) y planes especiales, para afrontar los riesgos a partir de los peligros concretos: químicos, nucleares, mercancías peligrosas, inundaciones o incendios forestales, entre otros.

La normativa española establece que "la protección civil es un servicio público, en cuya organización, funcionamiento y ejecución participan las diferentes administraciones públicas (...)". La Ley consta de un punto específico sobre los medios de comunicación donde describe la obligatoriedad de que colaboren con la administración. No se menciona la Web, pero el $M^{o}$ del Interior dispone de un sitio web 
parecido al de otros países occidentales, donde publica mapas y notas de prensa dirigidas a profesionales; tiene pocos apartados de divulgación de información para el público lego. En 2007 la DG de Protección Civil puso en funcionamiento una web destinada al público no especializado denominada Inforiesgos.

http://www.inforiesgos.es/

\section{Particularidades del caso catalán}

La organización de la protección civil en Catalunya depende de la $D G$ de Protección Civil y de la $D G$ de Prevención y Extinción de Incendios y Salvamentos, y es competencia orgánica del citado Departament d'Interior de la Generalitat. Según la Normativa Básica de Protección Civil (NBPC) del Estado español, cada comunidad autónoma debe crear y regular sus propios planes, en el caso catalán el Plan de Protección Civil de Catalunya (Procicat).

En lo referente a la comunicación y las tecnologías de la información, la Ley de Protección Civil de Catalunya, establece que el Gabinete de Información es el coordinador exclusivo dónde debe concentrarse toda la información que se genera. La normativa le atribuye la potestad de unificar los contenidos de las informaciones para evitar confusiones, contradicciones y establecer los modelos de comunicados, tanto a los medios de comunicación como a la población. La difusión de información a la opinión pública mediante las nuevas tecnologías de la información se estructura desde el sitio web del citado Departament d'Interior.

Esta web detalla los diferentes planes de emergencia, la organización del Departamento y los operativos que han de asumir la gestión. Contiene información técnica y legislativa, aunque poco divulgativa.

http://www.gencat.cat/interior/ emergencies/index.htm

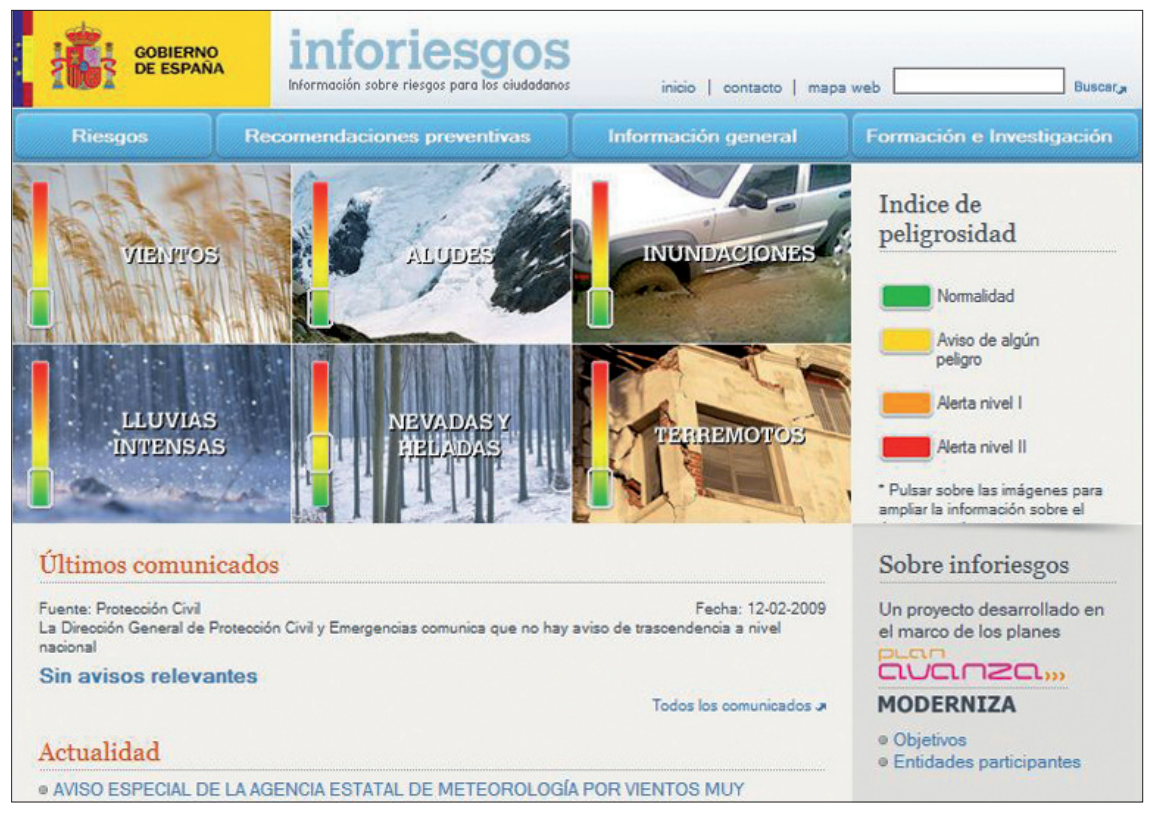

Imagen 1. Web de la Dirección General de Protección Civil y Emergencias para consultas del público

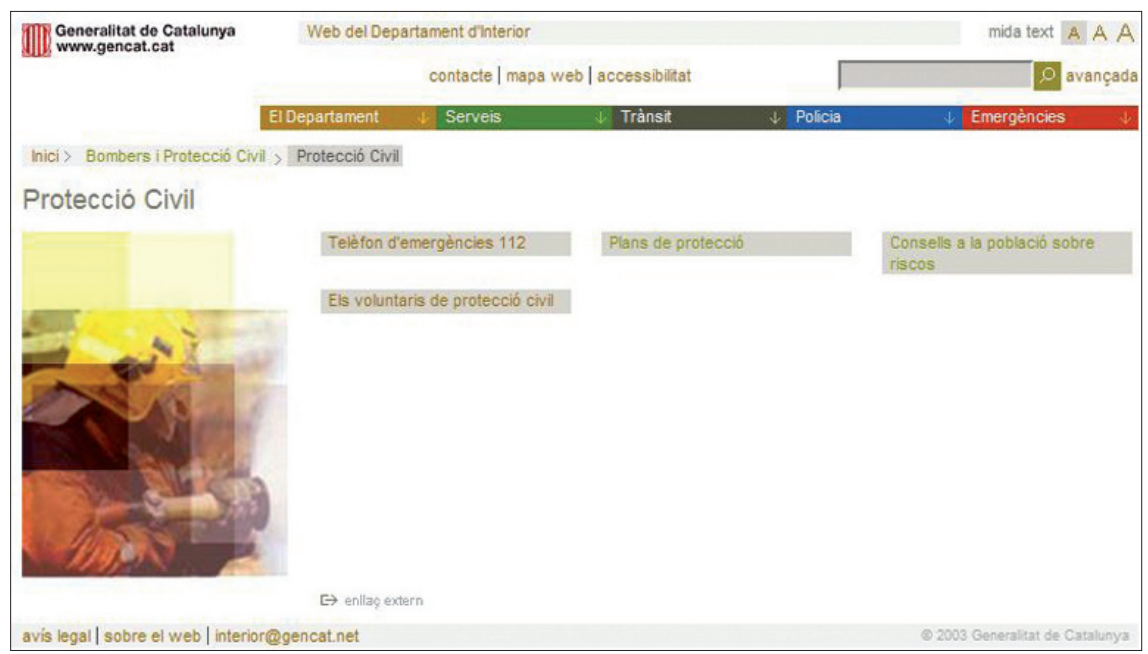

Imagen 2. Sitio web de emergencias de la Generalitat de Cataluña

\subsection{Experiencias internacionales}

La gestión de emergencias en los EUA se estructura a partir de la Federal Emergency Management Agency (FEMA), de la cual dependen numerosas agencias adscritas al departamento federal del interior, Department of Homeland Security, creado en 2003. A raíz de los atentados del 11 de septiembre de 2001, y tras la creación de este departamento, se han puesto al alcance de los ciudadanos dos webs que dan consejos en caso de emergencias: Ready America (versión en inglés) y Listo.Gov (versión en español). http://www.ready.gov/

http://www.listo.gov

En estos sitios web se pueden encontrar desde las instrucciones para un plan familiar de emergencia, hasta consejos prácticos como qué productos se deben tener almacenados permanentemente, hacia dónde hace falta huir y qué aparatos se deben tener encendidos, entre otros. Además, el propio FEMA ha creado una página para niños, FEMA for Kids.

En Europa gran parte de los países cuentan con direcciones de protección civil, como el organismo francés Direction de la Défen- 


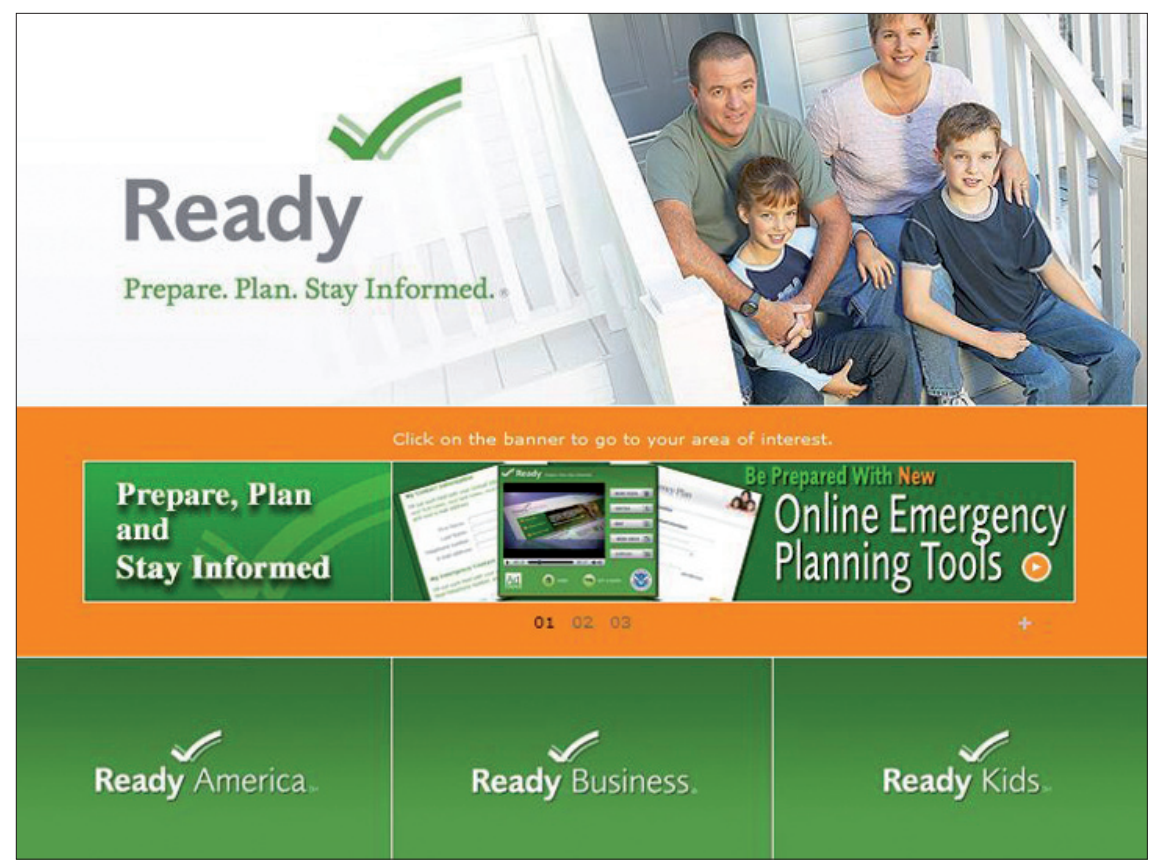

Imagen 3. Web que facilita consejos en caso de emergencia en los EUA. Destaca la minuciosidad de su información.

se et de la Sécurité Civiles, que depende del ministerio del interior francés.

Francia es pionera en la introducción de las nuevas tecnologías en la gestión de las emergencias, y tanto en la vertiente operativa como en la informativa se han planteado iniciativas para minimizar los riesgos, como el portal Prevéntion des Risques Majeurs (Prim).
La iniciativa forma parte del proyecto del gobierno francés para la sociedad de la información que en el caso de los riesgos ha llevado a cabo el Ministère de l'Aménagement du Territoire et de l'Environnement.

http://www.prim.net

El Reino Unido gestiona las crisis desde el Civil Contingencies Secretariat (CCS), que depende de

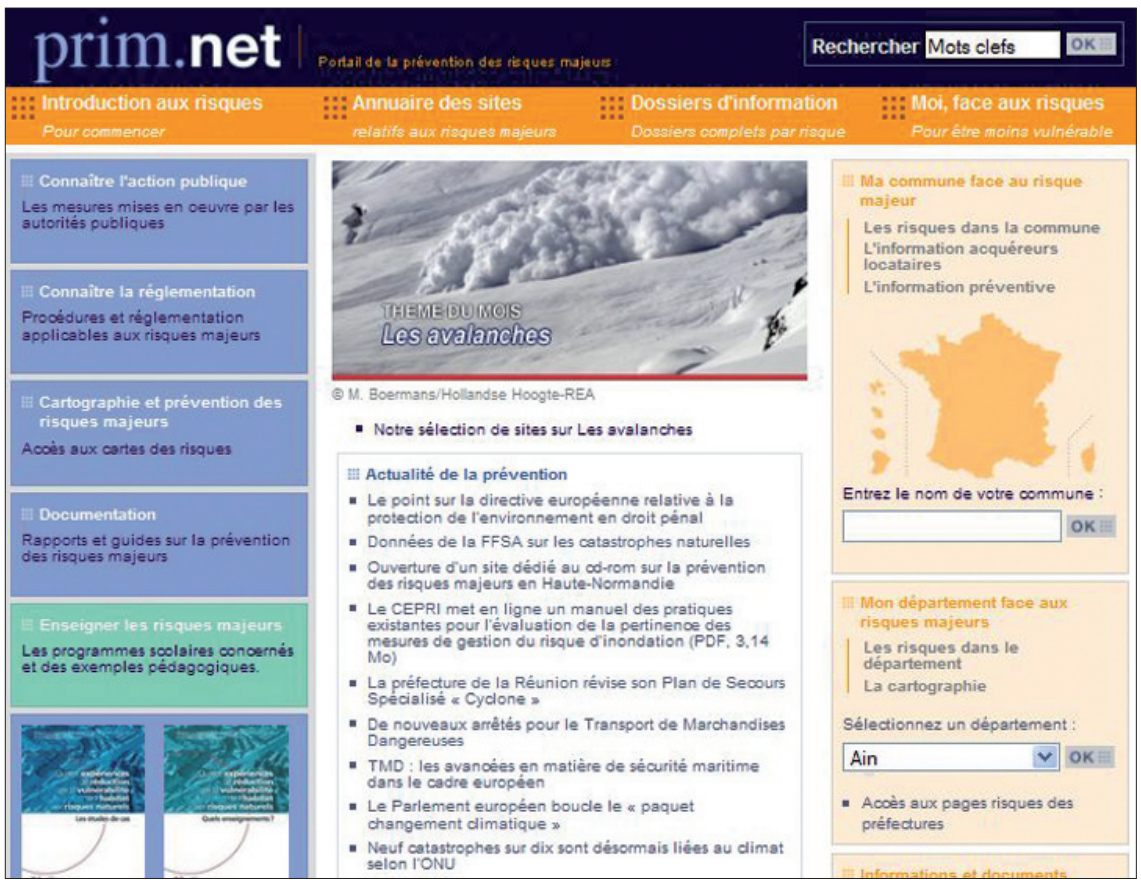

Imagen 4. Portal del gobierno francés sobre prevención de riesgos
The Cabinet Office, administración que ha creado el proyecto Preparing for Emergencies, dedicado a explicar a la población todo tipo de contingencias y su prevención o minimización. Además los británicos cuentan con The Emergency Planning College, un centro que depende del CCS y forma profesionales para la prevención, organizando foros y debates sectoriales, como el Media Emergency Forum $\& R M E F$, una experiencia innovadora que hace posible poner sobre la mesa las relaciones entre periodistas y administración en caso de desastre.

\section{Comparación de 5 webs sobre emergencias}

Todos los análisis se llevaron a cabo durante el mes de febrero y el día 2 de marzo de 2009.

El acceso de la totalidad de la población a la información de emergencia y riesgo es sin duda un objetivo prioritario. Por ello, en primer lugar se ha evaluado la accesibilidad de estos sitios web, es decir, el nivel de adecuación de sus contenidos a los usuarios con necesidades especiales (con deficiencias visuales, auditivas, o motrices, o que utilicen tecnologías de capacidad limitada). Como es sabido, existen tres niveles de adecuación: A, doble A (AA), y triple A (AAA) en función del grado de cumplimento de las reglas establecidas por la Iniciativa para la Accesibilidad Web (WAI) del World Wide Web Consortium (W3C).

A. Requisitos de prioridad 1: los que el sitio web "tiene" que satisfacer para que las personas con necesidades especiales puedan tener acceso a la información.

AA. Requisitos de prioridad 2: los que la web "debe" cumplir

AAA. Requisitos de prioridad 3: los que el sitio web "puede" cumplir para optimizar la accesibilidad de sus usuarios. 


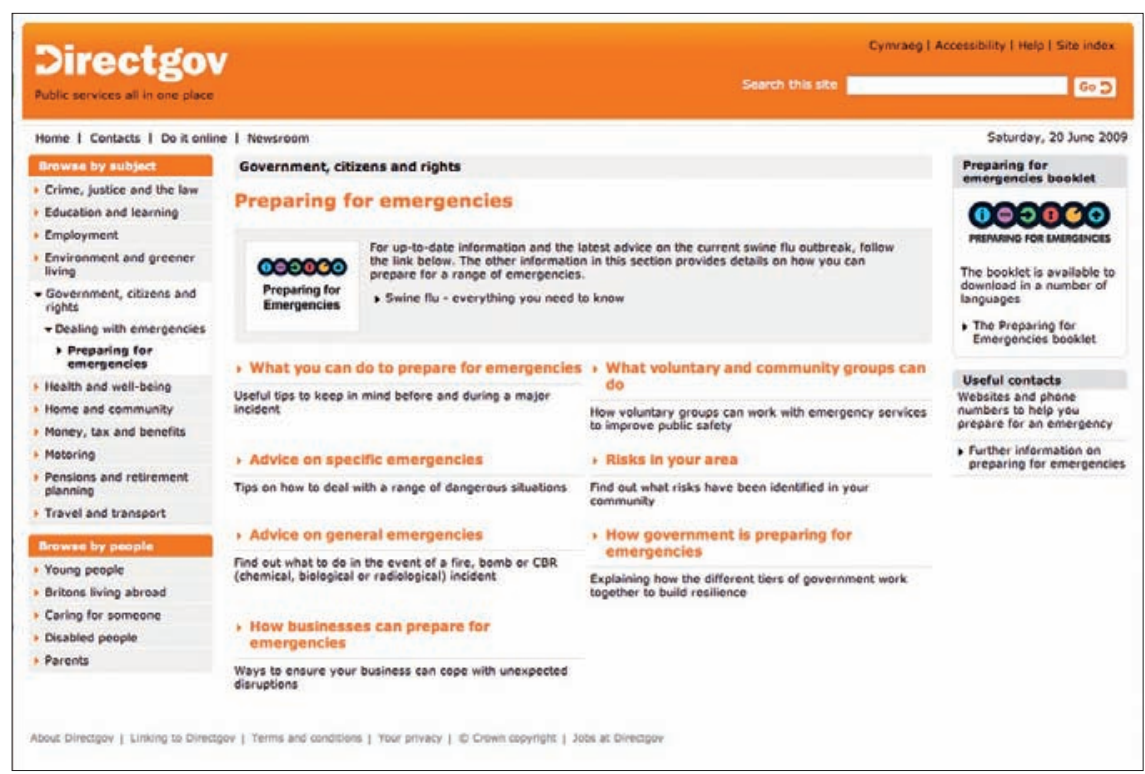

Imagen 5. Página de emergencias de Reino Unido.

Idealmente, un organismo público que pretenda llegar a la mayor cantidad posible de ciudadanos debería confeccionar su web conforme a un nivel AAA.

En la tabla 2 se muestran los resultados del análisis de la accesibilidad de las webs de emergencia, con el número de errores (detectados automáticamente) en cada sitio y para cada nivel de adecuación.

Es sorprendente que sólo uno de los cinco sitios webs no tenga errores de prioridad 1. Sólo Preparing for Emergencies (UK) cumple los requisitos mínimos para permitir el acceso de personas con necesidades especiales a sus contenidos, si bien el resto de webs cometen muy pocos errores de prioridad 1 , y les resultaría muy fácil corregirlos para conseguir el nivel de adecuación A.

\section{"Cuatro de las cinco webs analizadas podrían conseguir el nivel A con una pequeña mejora de su código fuente"}

Ninguna de las webs tendría un nivel de adecuación AA, aunque de nuevo, y para casi todos los casos, no necesitarían hacer un gran esfuerzo para conseguirlo. El único sitio que tendría dificultades para conseguir el nivel de adecuación AA sería el de la Generalitat de Catalunya, que presenta un número de errores muy elevado. El nivel AAA sólo lo cumple Preparing for Emergencies (UK), aunque para conseguirlo previamente tendría que corregir los errores de prioridad

\begin{tabular}{|l|c|c|c|c|}
\hline \multirow{2}{*}{ Sitio } & \multicolumn{4}{|c|}{$\begin{array}{c}\text { Número de errores de accesibilidad } \\
\text { (mediante el Test de Accesibilidad Web) }\end{array}$} \\
\cline { 2 - 5 } & Prioridad 1 & Prioridad 2 & Prioridad 3 & Total \\
\hline Inforiesgos & 1 & 12 & 2 & 15 \\
\hline $\begin{array}{l}\text { Emergencias Generalitat } \\
\text { de Catalunya }\end{array}$ & 1 & 201 & 12 & 214 \\
\hline Ready.gov & 2 & 11 & 3 & 16 \\
\hline Prim.net & 7 & 8 & 2 & 17 \\
\hline $\begin{array}{l}\text { Preparing for } \\
\text { emergencies }\end{array}$ & 0 & 9 & 0 & 9 \\
\hline
\end{tabular}

Tabla 2. Número de errores de accesibilidad
2. El resto de las webs nuevamente cometen un reducido número de errores de prioridad 3 que, como en los casos anteriores, son fácilmente solucionables. El segundo aspecto analizado es la visibilidad de la página principal. Cuanto mayor sea la visibilidad de un sitio web más fácil será encontrarlo (principalmente mediante el uso de buscadores). Se han utilizado las siguientes medidas:

- PageRank (visibilidad) según Google: esta medida va de 0 a 10 , siendo 10 la puntuación máxima.

- TrafficRank (popularidad) según Alexa: muestra la posición que ocupa la página analizada en el ranking mundial de las webs más visitadas que elabora Alexa. La puntuación máxima es 1, es decir, ser la página más popular (visitada) del mundo. Una página será más popular cuanto menor sea su número de orden en la clasificación TrafficRank.

- Número de enlaces que recibe cada sitio web según Yahoo: cuantos más enlaces recibe una web, más probable es que aparezca entre los primeros resultados de un motor de búsqueda.

- Número de webs indexadas por Yahoo de cada sitio web: esta medida nos indica cuántas páginas de un sitio web pueden ser recuperadas por un buscador. De nuevo es muy positivo tener un elevado número de páginas indexadas.

La tabla 3 muestra los resultados obtenidos.

Estableciendo una correlación entre los resultados obtenidos en las diferentes medidas, podemos determinar el siguiente ranking en función de la visibilidad y popularidad de las webs:

\section{Ready.gov}

\section{Prim.net}

\section{Preparing for emergencies}

\section{Inforiesgos}

5. Emergencias Generalitat de 


\begin{tabular}{|l|c|r|r|r|}
\hline \multirow{2}{*}{\multicolumn{1}{|c|}{ Sitio }} & \multicolumn{4}{|c|}{ Medidas de visibilidad y popularidad } \\
\cline { 2 - 5 } & $\begin{array}{c}\text { Page- } \\
\text { Rank } \\
\text { (Google) }\end{array}$ & $\begin{array}{c}\text { Traffi- } \\
\text { CRank } \\
\text { (Alexa) }\end{array}$ & $\begin{array}{c}\text { Enlaces } \\
\text { recibidos } \\
\text { (Yahoo) }\end{array}$ & $\begin{array}{c}\text { Páginas } \\
\text { indexa- } \\
\text { das } \\
\text { (Yahoo) }\end{array}$ \\
\hline Inforiesgos & 7 & 2.332 .150 & 918 & 346 \\
\hline $\begin{array}{l}\text { Emergencias Generalitat } \\
\text { de Catalunya }\end{array}$ & 5 & No alcanza & 846 & 80 \\
\hline Ready.gov & 9 & 122.871 & 133.015 & 1.366 \\
\hline Prim.net & 7 & 325.135 & 11.023 & 33.270 \\
\hline Preparing for emergencies & 7 & 2.252 .035 & 136.629 & 176 \\
\hline
\end{tabular}

Tabla 3. Resultados del análisis de visibilidad

\section{Catalunya}

Destacan los resultados negativos obtenidos por las webs españolas.

El tercer aspecto valorado ha sido la calidad del código fuente de la página principal, mediante el validador del $W 3 C$. En la tabla 4 podemos ver el número de errores que presenta cada sitio web.

En cuarto lugar se ha valorado la presencia de buscadores internos y de opciones de búsqueda avanzada, así como si existe alguna organización taxonómica de los contenidos o, en su defecto, un mapa de la web. Los resultados pueden verse en la tabla 5.

En cuanto al contenido, los aspectos estudiados han sido:

- Audiencia. Se ha valorado atendiendo a dos criterios:

a) contenidos para distintos públicos. Esta información ha debido inferirse por inspección de los contenidos;

b) navegación, es decir, si el menú de la página principal permite acceder a secciones específicas dirigidas a diferentes tipos de usuarios. Típicamente, se trataría de seis tipos: público general, expertos en la materia, voluntarios, empresas, niños y autoridades.

Los resultados pueden verse en las tablas $6 a$ y 6 b.

- Tipo de riesgos. Se trató de determinar sobre cuáles de los riesgos más habituales facilitaban ban enormemente. Por su clarida destaca Ready.gov, y por su cantidad Prim.net. En líneas generales la información ofrecida por los sitios webs extranjeros es de mayor calidad (y en mayor cantidad) que los españoles. Especialmente pobres son los contenidos de la web de emergencias de la Generalitat de Catalunya. También es interesante comprobar cómo las webs de EUA y Reino Unido contienen informa- ción sobre cómo actuar ante un hipotético ataque terrorista.

- Canales de comunicación: se ha estudiado qué canales de comunicación complementarios/alternativos a la propia web se ponen a disposición de los usuarios. Pueden verse detallados en la tabla 7 .

- Consejos a la población: todas las webs proporcionan consejos a la población sobre qué hacer, dónde estar, cómo protegerse, o cuáles son los suministros que deben poseerse ante una situación de emergencia concreta. No obstante, de la misma forma que ocurría con el tratamiento de los distintos tipos de riesgos, la cantidad y la calidad de la información suministrada difiere. La web que mejores recomendaciones da a la población es Ready.gov, caracterizada por su completitud, claridad y concisión.

\section{"La integración de las nuevas tecnologías en la comunicación institucional de emergencias es importante para llegar a toda la población"}

\begin{tabular}{|l|c|c|}
\hline \multicolumn{1}{|c|}{ Sitio } & $\begin{array}{c}\text { Número de } \\
\text { errores }\end{array}$ & $\begin{array}{c}\text { Tipo de documento } \\
\text { (Doctype) }\end{array}$ \\
\hline Inforiesgos & 21 & xhtml 1.0 Transitional \\
\hline $\begin{array}{l}\text { Emergencias Generalitat de } \\
\text { Catalunya }\end{array}$ & 92 & html 4.01 Transitional \\
\hline Ready.gov & 18 & xhtml 1.0 Transitional \\
\hline Prim.net & 25 & xhtml 1.0 Strict \\
\hline Preparing for emergencies & 7 & xhtml 1.0 Transitional \\
\hline
\end{tabular}

Tabla 4. Número de errores en el código

\begin{tabular}{|l|c|c|c|}
\hline \multicolumn{1}{|c|}{ Sitio } & $\begin{array}{c}\text { Búsqueda } \\
\text { simple }\end{array}$ & $\begin{array}{c}\text { Búsqueda } \\
\text { avanzada }\end{array}$ & $\begin{array}{c}\text { Mapa } \\
\text { de la web }\end{array}$ \\
\hline Inforiesgos & sí & no & sí \\
\hline $\begin{array}{l}\text { Emergencias Generalitat de } \\
\text { Catalunya }\end{array}$ & no & no & no \\
\hline Ready.gov & sí & sí & sí \\
\hline Prim.net & sí & no & sí \\
\hline Preparing for emergencies & sí & no & sí \\
\hline
\end{tabular}

Tabla 5. Puntos de acceso a la información 


\begin{tabular}{|l|c|c|c|c|c|c|}
\hline \multicolumn{1}{|c|}{ Sitio } & $\begin{array}{c}\text { Público } \\
\text { general }\end{array}$ & Expertos & Voluntarios & Empresas & Niños & Autoridades \\
\hline Inforiesgos & + & + & - & - & - & - \\
\hline $\begin{array}{l}\text { Emergencias Generalitat de } \\
\text { Catalunya }\end{array}$ & + & - & - & + & - & + \\
\hline Ready.gov & + & - & - & + & + & - \\
\hline Prim.net & + & + & - & - & + & + \\
\hline Preparing for emergencies & + & + & + & + & - & + \\
\hline
\end{tabular}

Tabla 6a. Tipos de contenidos segmentados por posibles audiencias

\begin{tabular}{|l|c|c|c|c|c|c|c|}
\hline \multicolumn{1}{|c|}{ Sitio } & $\begin{array}{c}\text { ¿Presenta } \\
\text { navegación } \\
\text { por } \\
\text { audiencias? }\end{array}$ & $\begin{array}{c}\text { Público } \\
\text { general }\end{array}$ & Expertos & Voluntarios & Empresas & Niños & Autoridades \\
\hline Inforiesgos & no & & & & & & \\
\hline $\begin{array}{l}\text { Emergencias Generalitat } \\
\text { de Catalunya }\end{array}$ & no & & & & & & \\
\hline Ready.gov & sí & + & - & - & + & + & + \\
\hline Prim.net no & & & & & & + \\
\hline $\begin{array}{l}\text { Preparing for } \\
\text { emergencies }\end{array}$ & sí & + & - & + & + & - & + \\
\hline
\end{tabular}

Tabla 6b. Navegación por audiencias y tipos de audiencias contempladas en la navegación (labeling) de cada sitio

\begin{tabular}{|l|c|c|c|c|c|}
\hline \multicolumn{1}{|c|}{ Sitio } & Teléfono & $\begin{array}{c}\text { Medios de } \\
\text { comunicación }\end{array}$ & $\begin{array}{c}\text { Centros de } \\
\text { información } \\
\text { pública }\end{array}$ & $\begin{array}{c}\text { RSS / } \\
\text { ATOM }\end{array}$ & email \\
\hline Inforiesgos & sí & no & no & no & sí \\
\hline $\begin{array}{l}\text { Emergencias } \\
\text { Generalitat } \\
\text { de Catalunya }\end{array}$ & sí & no & no & no & no \\
\hline Ready.gov & sí & sí & no & no & si \\
\hline Prim.net & no & sí & no & sí & sí \\
\hline $\begin{array}{l}\text { Preparing for } \\
\text { emergencies }\end{array}$ & sí & sí & no & no & si \\
\hline
\end{tabular}

Tabla 7. Medios de comunicación complementarios

\section{Conclusiones y recomendaciones}

1. Los cinco casos analizados muestran las potencialidades de los sistemas de información basados en la Web para los procesos de comunicación de riesgo porque ayudan a integrar y gestionar mejor la información.

La integración de las nuevas tecnologías en la comunicación institucional de emergencias es importante para llegar más rápida y universalmente a la población. Pueden ayudar a prevenir situaciones de riesgo y mejoran la gestión de las emergencias. La población de países como los considerados, hace tiempo que está acostumbrada a utilizar la web para obtener toda clase de informaciones, y la incorporación de internet en los hogares (no digamos en las empresas) es casi universal. No es una hipótesis arriesgada suponer que en caso de emergencia una buena parte de la población buscará información en la web de forma pro activa.

2. En algunos de los casos analizados se han detectado deficiencias en el nivel de accesibilidad, un aspecto clave de cualquier web dirigida a facilitar información de riesgo y emergencia al público general.

Se calcula que, en cualquier país, entre un 10 y un $20 \%$ de sus ciudadanos tiene algún tipo de discapacidad. La información sobre emergencias debe llegar al mayor número posible de personas y por tanto es poco justificable obviar una audiencia del 10-20\%. Desafortunadamente, ninguno de los sitios web analizados cumple los requisitos deseables en esta materia. Pero, mientras en los sitios de EUA, Reino Unido, Francia y España el número de errores es relativamente pequeño, es grave el caso de la web de emergencias de la Generalitat de Cataluña. Este aspecto es destacable de forma negativa cuando tomamos en consideración la legislación española. Concretamente, el Real Decreto 1494/2007 de 12 de noviembre establece claramente que las webs de las instituciones públicas españolas deben cumplir obligatoriamente los niveles de prioridad 1 y 2 a partir del 31 de diciembre de 2008. Por tanto, podemos afirmar que ninguna de las webs españolas analizadas cumple con los criterios de accesibilidad mínimos fijados por la ley.

3. En España, y más concretamente en Catalunya, el uso de sis- 
temas de información basados en la web no se ha puesto en práctica suficientemente si los comparamos con los otros modelos analizados.

La información publicada por las administraciones catalana $\mathrm{y}$ española es confusa y poco estructurada para los públicos legos, principales destinatarios de esta información. La ausencia de una navegación adaptada al tipo de audiencia que visita la web es un claro indicador de su poca elaboración, que contrasta con la organización y claridad de las webs extranjeras objeto de estudio. La publicación de materiales en la Red parece más orientada a cumplir normativas que a ser una herramienta de formación o un elemento eficaz de comunicación. Por otra parte, las administraciones españolas mantienen un cierto retraso en la comunicación directa con el ciudadano.

4. EUA, Francia y Reino Unido son algunos de los países con modelos de gestión comunicativa de las emergencias más innovadores.

En la vanguardia de la gestión de emergencias se encuentran los EUA. Uno de los pilares del sistema comunicativo americano de emergencias es el sitio web que hemos considerado en este estudio, donde se pueden encontrar desde las instrucciones para un plan familiar de emergencia, hasta consejos de orden práctico.

El gobierno francés también apuesta por una política proactiva en la gestión comunicativa del riesgo similar al modelo americano. En internet, el gobierno galo ha hecho una gran apuesta a partir del portal Prim.net, donde se puede encontrar todo tipo de información para la población en referencia al tratamien- to de las emergencias que puedan acaecer en territorio francés. En el mismo sentido, el gobierno británico ha creado el sitio web Preparing for Emergencies, dedicado a explicar a un amplio espectro de la población todo tipo de contingencias y su prevención.

Probablemente, constituyen tres de los mejores modelos tanto para los gobiernos autonómicos con competencias en emergencias, como para el gobierno de España a la hora de realizar un estudio de benchmarking en este campo. Además se considera como altamente positivo el uso de diferentes idiomas, como en el caso de la web americana que cuenta con versión en inglés y versión en español.

\section{Notas}

1. Se entiende como web de emergencias por analogía a protección civil. Ley 2/1985, de 21 de enero, sobre Protección Civil (BOE n. 22 de 25-01-85). La protección civil es "identificada doctrinalmente como protección física de las personas y de los bienes, en situación de grave riesgo colectivo, calamidad pública o catástrofe extraordinaria, en la que la seguridad y la vida de las personas pueden peligrar y sucumbir masivamente, la protección civil constituye la afirmación de una amplia política de seguridad, que encuentra su fundamento jurídico, dentro de la Constitución, en la obligación de los poderes públicos de garantizar el derecho a la vida y a la integridad física, como primero y más importante de todos los derechos fundamentales (art. 15), en los principios de unidad nacional y solidaridad territorial (art. 2), y en las exigencias esenciales de eficacia y coordinación administrativa (art. 103)".

\section{Bibliografía}

Beck, Ulrich. La sociedad del riesgo global. Madrid: Siglo Veintiuno de España Editores, 2002.

Bennet, Peter; Calman, Kenneth (eds.). Risk communication and public health. New York: Oxford University Press, 1999.

Burkhart, Ford N. Media, emergency warnings, and citizen response. Boulder Colorado: Westview, CO, 1991.

Burnett, John J. "A strategic approach to manage crises". Public relations review, 1998, v. 24, n. 4 , pp. $475-488$.

Castelló, Enric; Domingo, David. "L'ús de les TIC en la comunicació de risc i la gestió d'emergències: oportunitats i límits". En: Farré, J.; Fernández-Cavia, J. (eds.) Comunicació i risc petroquímic a Tarragona. De les definicions a les pràctiques institucionals. Tarragona: Publicacions URV-Cossetània, 2007.

Codina, Lluís. "Posicionamiento Web: conceptos y ciclo de vida". Hipertext.net, 2004, n. 2. http://www.hipertext.net

Committee on Risk Perception and Communication, National Research Council. Improving risk communication. Washington, DC: National Academy Press, 1989

Farré, Jordi. "Comunicación de riesgo y espirales del miedo". Comunicación y sociedad, 2005, n. 3, pp. 95-119. Nova època.

Gil-Calvo, Enrique. El miedo es el mensaje. Riesgo, incertidumbre y medios de comunicación. Madrid: Alianza Editorial, 2004.

Murdock, Graham; Horlick-Jones, Tom; Petts, Judith. Social amplification of risk: the media and the public. Contract research report, Health \& Safety Executive (HSE), 2001.

Pauchant, Thierry C.; Mitroff, Ian I. "Crisis prone versus crisis avoiding organizations: Is your company's culture its own worst enemy in creating crises?". Industrial crisis quarterly, 1991, v. 2, n. 1, pp. 53-63.

Reinhardt, Claudia. "How to handle a crisis". Public relations journal. Association for Education in Journalism and Mass Communication, 1987 , v. 43 , n. 11 , pp. $43-44$

Starr, Chauncey. "Social benefit versus technological risk". Science, 1969, n. 165, pp. $1232-$ 1238 .

Voces-Merayo, Ramón. “¿Por qué la web debe ser accesible?” Hipertext.net, n. 5, 2007.

http://www.hipertext.net

Carles Pont-Sorribes, Lluís Codina, Rafael Pedraza-Jimenez.

Universitat Pompeu Fabra.

carles.pont@upf.edu

lluis.codina@upf.edu

rafael.pedraza@upf.edu 
